\title{
Attitudes of expectant fathers in the setting of teenage pregnancy towards breastfeeding - a mixed methods study
}

\begin{abstract}
Background: Breastfeeding rates in the setting of teenage pregnancy are poor and the attitudes of fathers poorly documented. The aim was to explore the attitudes of fathers in the setting of teenage pregnancy towards breastfeeding.

Methods: Observational study of 50 expectant fathers in the setting of teenage pregnancy (teenage group) and 100 expectant fathers not in the setting of teenage pregnancy (comparison group), but living within the same metropolitan healthcare region. Fathers were enrolled in the larger Australian Father's Study and were recruited from antenatal clinics and community settings. Researchers administered demographic, attitudinal and role in decision-making questionnaires.

Results: Teenage group fathers were younger, more likely to be born in Australia, have $<12$ years of education, be unemployed and smoke (all $p<0.05$ ). They had a strong preference for natural birth (78\%) without surgical or analgesic intervention $(74 \%)$. Despite this, only $22 \%$ wanted their partner to breastfeed. Three themes emerged in fathers with positive attitudes: breastfeeding was natural, best for baby and convenient. Five themes emerged in fathers with negative attitudes: breast ownership, messy and smelly, deprives father of a role, myths and worse for baby.

Conclusion: Fathers in the setting of teenage pregnancy desire natural childbirth but their attitudes towards breast feeding are poor. Five negative themes were identified. Educating fathers may support teenage mothers who wish to breastfeed.

Trial registration: The Australian Father's Study is registered at the Australasian Clinical Trials Registry with the number ACTRN12613001273774. The trial was registered on 19 November 2013. The trial website is located at http:// australianfathersstudyresearchtrial.weebly.com.
\end{abstract}

Keywords: breastfeeding, teenage pregnancy, mixed methods study, education, attitudes, teenage fathers, pregnancy

\author{
Volume 4 Issue I - 2018 \\ Danielle Lam,' Rodney W Petersen, ${ }^{2}$ Julie A \\ Quinlivan ${ }^{3}$ \\ 'Department of Obstetrics and Gynaecology, Joondalup Health \\ Campus, Australia \\ 'Women's and Babies Service,Women's and Children's Hospital, \\ Australia \\ ${ }^{3}$ Institute for Health Research, University of Notre Dame \\ Australia, Australia
}

Correspondence: Julie A Quinlivan, Institute for Health Research, University of Notre Dame Australia, Fremantle, 6160, WA, Australia, Tel 6189409631 ,

Email Julie.Quinlivan@nd.edu.au

Received: February 24, 2017 | Published: January 10, 2018

\section{Implications statement}

Fathers in the setting of teenage pregnancy may have a negative attitude towards breastfeeding. Breastfeeding education for fathers is desirable to ensure they are aware of the short-term and long-term health benefits for infant and mother.

\section{Background}

Teenage pregnancy is associated with poor birth outcomes and lower rates of breastfeeding. ${ }^{1-4}$ However, most studies in the setting of teenage pregnancy are gender biased with a significantly lower rate of reports on the attitudes and roles of the fathers during antenatal, birth and post-natal periods. ${ }^{5,6}$

Contemporary Western society has grown to accept and expect fathers' involvement in the antenatal period and birthing process of their offspring. Fathers are also recognised to share domestic responsibilities, which encompass taking on an active role in caring for and the upbringing of their children. ${ }^{5,7}$ Of the limited research centred on fathers in the setting of teenage pregnancy, it has been noted that teenage fatherhood is associated with high levels of psychological distress and adverse social outcomes such as low socioeconomic status, incomplete education, and employment instability. ${ }^{8}$ Fathers in the setting of teenage pregnancy find themselves facing responsibility, expectation and stress with fewer life skills to cope with these demands.

Previous studies have reported that the prospect of fatherhood is a powerful motivator for positive change in teenage fathers. ${ }^{1,8}$ Paternal involvement and support in the setting of teenage pregnancy has also been identified as a strong factor in the improvement of teenage pregnancy birth outcomes and in the subsequent development and behaviour of offspring. ${ }^{3,4,9,10}$

The World Health Organisation recommends exclusive breastfeeding for all infants from birth to six months of age. ${ }^{11}$ The biopsychosocial benefits for mother and child are well evident in numerous studies and hence breastfeeding is a healthcare initiative that is widely advocated. ${ }^{2,11}$ The benefits of breastfeeding include reduced shortterm and long-term physical and mental health morbidities for the infant, promotion of attachment between mother and child, and lower economic cost. ${ }^{10,12}$ However, breastfeeding has been identified as a practice that is poorly initiated and continued by teenage mothers. ${ }^{3,4}$ Social perception, expectations and lack of breastfeeding education 
are factors reported to contribute to low rates of breastfeeding. ${ }^{3,4}$ One American study noted that fathers' support of breastfeeding (or lack of) was a significant factor in a mother's decision to breastfeed. ${ }^{13}$ The aim of this study was to explore the attitudes of fathers in the setting of teenage pregnancy towards breastfeeding.

\section{Methods}

\section{Type of study, ethics, consent and permission}

This mixed methods study was a sub-study within the Australian Father's Study. The Australian Father's Study is a longitudinal study addressing father's attitudes towards antenatal, birth and postnatal care. The Australian Father's Study has institutional ethics committee approval (Primary ethics committee: Joondalup Health Campus Approval number 1301). Individual informed consent was obtained from each participant.

\section{Study population}

The study population consisted of 50 expectant fathers whose pregnant partner was aged less than 20years (teenage group fathers). Fathers in this group were not all themselves teenagers, as the mean age of fathers was 2.1years older than the pregnant mother.

The comparison group were expectant fathers recruited from the same metropolitan health service region, whose pregnant partner was not a teenager (comparison group fathers). The comparison group fathers had demographic features similar to the wider population of expectant Australian fathers in terms of age, religious belief, years of education, employment and first time fatherhood status. ${ }^{14}$

Fathers were recruited by research staff and completed questionnaires addressing demographic variables, attitudes and roles in decision-making. Quantitative and qualitative data were extracted from completed questionnaires.

\section{Inclusion and exclusion criteria}

Expectant fathers were recruited via the pregnant mother, so the study was only able to include men where the mother acknowledged the man to be the father of the baby and gave consent for the father to participate. Fathers in the setting where pregnancy was complicated by a known fetal anomaly were excluded. Fathers with insufficient English to complete questionnaires were also excluded from this substudy.

\section{Variables to be measured and examined}

The antenatal questionnaire was completed in the third trimester. It consisted of qualitative and quantitative components. The variables measured and examined through the questionnaire were collected in three broad areas: background demographics, preferences and attitudes, and role in decision-making. These variables were used as a framework for analysis of the primary outcome.

\section{Primary hypothesis and sample size}

The primary hypothesis was that teenage group fathers would have a poorer attitude towards breastfeeding than the comparison group fathers. Assuming $50 \%$ of teenage group fathers had a positive attitude, compared to $80 \%$ of comparison group fathers, a sample of 45 teenage group fathers provided $80 \%$ power with an error of 0.05 to detect this difference. The study recruited 50 teenage group fathers to ensure a sufficient sample, assuming incomplete data might be available from some questionnaires. It was decided to enrol a comparison group of fathers twice the size of the teenage group to increase study power.

\section{Statistical analysis}

Data was entered onto a dataset using Minitab ${ }^{\circledR}$ (version 16, University of Melbourne). A descriptive analysis was used for the quantitative data. In order to analyze for differences in responses between teenage group fathers and comparison group fathers, the significance (p) of the differences was determined by a chi-square test $\left(\chi^{2}\right)$ for independence. Fisher Exact test was utilized where cell size was less than five. A p-value of 0.05 was set for rejection of the null hypothesis. A student $t$ test was used to compare continuous data.

For the qualitative data in the comments section of the questionnaire, an inductive content analysis was performed in accordance with methodology described by Elo \& Kyngäs. ${ }^{15}$ The written comments were independently read by the principal researchers and an abstraction process used to summarize and conceptualize the overall meaning and implications of the comments. Open coding was performed to maximize the number of headings in order to describe all aspects of the content. Both quantitative and qualitative aspects of the data were integrated for data interpretation.

\section{Results}

The demographic characteristics of the study participants are summarised in Table 1. Teenage group fathers were significantly younger (teenage 21years versus comparison 32years, $\mathrm{p}<0.0001$ ). They were more likely to have been born in Australia (teenage $92 \%$ versus $68 \%, p<0.0001$ ).A higher prevalence of teenage group fathers were of Aboriginal or Torres Strait Islander origin (teenage $18 \%$ versus $3 \%, \mathrm{p}<0.0001)$. Of note, only $32 \%$ of teenage group fathers were living with their partner $(32 \%$ versus $90 \%, \mathrm{p}<0.0001)$. Less teenage group fathers were educated beyond 12 years (teenage $2 \%$ versus $53 \%, \mathrm{p}<0.0001)$. The rate of unemployment was higher in teenage group fathers (teenage $32 \%$ versus $5 \%, \mathrm{p}<0.0001$ ). The prevalence of smokers in the teenage fathers group was higher (teenage $38 \%$ versus $21 \%, \mathrm{p}<0.009$ ). Almost all teenage group fathers were first-time fathers (teenage $96 \%$ versus $52 \%, \mathrm{p}<0.0001$ ), and the rate of unplanned pregnancy was significantly higher (teenage $78 \%$ versus $19 \%, \mathrm{p}<0.0001)$.

Table 2 summarises the antenatal preferences and attitudes of expectant fathers. Teenage group fathers had a significantly higher preference for a natural or vaginal delivery (teenage $78 \%$ versus $45 \%$, $\mathrm{p}<0.0001)$. They also had a stronger preference for natural or no analgesia in labour (teenage $74 \%$ versus $36 \%, \mathrm{p}<0.0001$ ).

Attitudes towards Down syndrome screening and infant vaccination were similar compared to the comparison fathers. However, attitudes towards breastfeeding were significantly less positive. Only $30 \%$ of teenage group fathers had a positive attitude towards breastfeeding. Nearly half stated their attitude was negative (45\%). In contrast, $85 \%$ of the comparison group fathers identified a positive attitude towards breastfeeding $(\mathrm{p}<0.0001)$

Thirty-one of the 50 teenage group fathers wrote comments about breastfeeding. Three themes were identified in the comments made by teenage group fathers who had a positive attitude towards breastfeeding. These were that breastfeeding was a natural process, breastfeeding was best for the baby and convenient. Fathers believed breastfeeding was natural as babies were being provided with human milk. Comments included: 
i. "Breastfeeding is natural"

ii. "Babies should probably be breastfed as its (sic) human milk"

Table I Demographics of expectant fathers in the setting of teenage pregnancy compared to control

\begin{tabular}{|c|c|c|c|}
\hline & Control & Teenage & P-Value \\
\hline & $N=100$ & $\mathbf{N}=\mathbf{5 0}$ & \\
\hline \multicolumn{4}{|l|}{ Age } \\
\hline Mean (sd) & $31.8(3.1)$ & $21.2(1.2)$ & $<0.0001$ \\
\hline \multicolumn{4}{|l|}{ Country of birth } \\
\hline Australia & $68 \%$ & $92 \%$ & $<0.0001$ \\
\hline Elsewhere & $32 \%$ & $8 \%$ & \\
\hline $\begin{array}{l}\text { Aboriginal or torres strait } \\
\text { islander }\end{array}$ & $3 \%$ & $18 \%$ & $<0.0001$ \\
\hline \multicolumn{4}{|l|}{ Relationship status } \\
\hline Living with partner & $90 \%$ & $32 \%$ & $<0.000$ I \\
\hline Not living with partner & $10 \%$ & $68 \%$ & \\
\hline \multicolumn{4}{|l|}{ Education } \\
\hline$<12$ years & $7 \%$ & $24 \%$ & $<0.0001$ \\
\hline 12 years & $40 \%$ & $74 \%$ & \\
\hline$>12$ years & $53 \%$ & $2 \%$ & \\
\hline \multicolumn{4}{|l|}{ Employment } \\
\hline No & $5 \%$ & $32 \%$ & $<0.0001$ \\
\hline Yes- locally & $75 \%$ & $54 \%$ & \\
\hline Yes - FIFO & $20 \%$ & $14 \%$ & \\
\hline \multicolumn{4}{|l|}{ Hours employed } \\
\hline $0-15$ & $9 \%$ & $35 \%$ & $<0.0001$ \\
\hline $15-40$ & $23 \%$ & $61 \%$ & \\
\hline $40+$ & $68 \%$ & $4 \%$ & \\
\hline Smoker & $21 \%$ & $38 \%$ & 0.009 \\
\hline \multicolumn{4}{|l|}{ First time father } \\
\hline Yes & $52 \%$ & $96 \%$ & $<0.0001$ \\
\hline No & $48 \%$ & $48 \%$ & \\
\hline \multicolumn{4}{|l|}{ Pregnancy planning } \\
\hline Natural - planned & $70 \%$ & $22 \%$ & $<0.0001$ \\
\hline IVF - planned & $11 \%$ & $0 \%$ & \\
\hline Unplanned & $19 \%$ & $78 \%$ & \\
\hline
\end{tabular}

Fathers believed breastfeeding was better for the baby and would help the baby grow and develop. Interestingly the fathers universally referred to "they" or else named a specific older adult who had told them breastfeeding was better for the baby. Comments included:

i. "They say it's (sic) better for the baby."

ii. "My mum told me its (sic) supposed to help the baby".

iii. Fathers identified that breastfeeding was easier to manage compared to formulae feeding as bottles and formulae did not need to be carried about or stored. By example, fathers wrote: iv. "It's probably easier than carrying bottles around"

v. "She won't (sic) have to buy milk or carry it everywhere"

Table 2 Antenatal preferences and attitudes of expectant fathers in the setting of teenage pregnancy compared to control

\begin{tabular}{|c|c|c|c|}
\hline & Control & Teenage & P-value \\
\hline & $N=100$ & $\mathbf{N}=\mathbf{5 0}$ & \\
\hline \multicolumn{4}{|l|}{ Preferred birth } \\
\hline Natural/vaginal & $45 \%$ & $78 \%$ & $<0.0001$ \\
\hline $\mathrm{C} / \mathrm{S}$ & $25 \%$ & $2 \%$ & \\
\hline No preference & $30 \%$ & $20 \%$ & \\
\hline \multicolumn{4}{|c|}{ Preferred labour analgesia } \\
\hline Natural & $36 \%$ & $74 \%$ & $<0.0001$ \\
\hline Minor - gas or pethidine & $3 \%$ & $0 \%$ & \\
\hline Epidural & $20 \%$ & $0 \%$ & \\
\hline No preference & $41 \%$ & $26 \%$ & \\
\hline \multicolumn{4}{|c|}{ Attitude to down syndrome screen } \\
\hline Positive & $80 \%$ & $80 \%$ & 0.39 \\
\hline Neutral & $10 \%$ & $14 \%$ & \\
\hline Negative & $10 \%$ & $6 \%$ & \\
\hline \multicolumn{4}{|c|}{ Attitude to infant vaccination } \\
\hline Positive & $82 \%$ & $90 \%$ & 0.16 \\
\hline Neutral & $14 \%$ & $10 \%$ & \\
\hline Negative & $4 \%$ & $0 \%$ & \\
\hline \multicolumn{4}{|c|}{ Attitude to breastfeeding } \\
\hline Positive & $85 \%$ & $30 \%$ & $<0.0001$ \\
\hline Neutral & $12 \%$ & $25 \%$ & \\
\hline Negative & $3 \%$ & $45 \%$ & \\
\hline
\end{tabular}

Conversely, teenage group fathers who reported a negative view of breastfeeding identified five themes. These themes were: ownership of the breast, a belief that breastfeeding was messy and smelly, it deprived the father of a role, breastfeeding benefits were a myth, and breastfeeding was not best for the baby.

Teenage group fathers demonstrated a possessive attitude towards their partner's breasts. They were seen as an object that belonged to the father or the relationship. There were concerns expressed breastfeeding would detract from their enjoyment of the breast as a sexual object. Comments included:

i. "I don't want her showing her breasts off to other blokes".

ii. "I don't like the idea of breastfeeding, It takes away from us".

iii. "No way I want her breastfeeding. Her breasts will sag and leak. I wouldn't want to touch them"

iv. Some fathers commented on their concern breastfeeding would be messy and smelly. Comments included:

v. "Breastfeeding is messy. Doesn't milk leak everywhere"

vi. "It would smell up the bed" 
Some fathers commented that breastfeeding would prevent them from participating in the care of the baby. Their comments were almost competitive, as if the option to breastfeed denied them the opportunity to fulfil their own care provider goals. Comments included:

i. "If she breastfeeds then what do I do. I am looking forward to feeding the baby"

ii. "I want to feed the baby"

A small group of fathers stated the benefits of breastfeeding were a myth as they had not been breastfed and yet had grown up to be healthy. Comments included:

i. "They carry on that you should breastfeed but I wasn't and I'm okay"

ii. "I don't believe it is a micle (sic miracle) more like a myth"

The final negative theme centred on a belief that formulae was better than breastfeeding. The fathers were reassured by being able to observe milk volumes consumed by their baby and reassured by the advertising around the ingredients on formula packages. Comments included:

i. "If she feeds with a bottle you see what goes in the baby. That way you know the baby gets enough to eat"

ii. "The formula has a lot of things that make it better (sic healthier)"

\section{Discussion}

This Australian study has explored the attitude of fathers in the setting of a teenage pregnancy towards breastfeeding. Our study found poorer attitudes toward breastfeeding compared to older fathers. Several studies have reported on the factors that influence breastfeeding in the setting of teenage pregnancy. ${ }^{2-4,16,17}$ Factors that have been reported to positively contribute towards breastfeeding in teenage mothers include support from their significant others, education about the benefits of breastfeeding for their infant, and positive societal perceptions and attitudes towards women who breastfeed in a social setting. ${ }^{2-4,16}$ In contrast, a midwifery-based postnatal home visiting service did not facilitate prolongation of breastfeeding in teenage mothers. ${ }^{17}$

The qualitative analysis noted that fathers in the setting of teenage pregnancy with a positive attitude towards breastfeeding believed breastfeeding was "best for baby," This finding has been reported previously., ${ }^{3,4}$ Peer-aged-partners (of teenage mothers) who encouraged breastfeeding were mindful of the positive health benefits for the infant. ${ }^{3}$ These fathers were aware of the benefits of breastfeeding and were more likely to support and encourage their partners to breastfeed. Fathers in the setting of teenage pregnancy with a negative attitude towards breastfeeding displayed possessive attitudes towards their partner's breasts, were concerned about being deprived of a role in caring for their baby and perceived breastfeeding as messy and smelly. They also lacked education about benefits of breastfeeding.

A Western Australian study by Scott et al. ${ }^{4}$ highlighted the theme of sexual versus functional role of the breast and lack of fathers' support as a barrier towards breastfeeding in teenage mothers. Most young men in their study expressed embarrassment at seeing a woman breastfeed and experienced a sense of "ownership of their partners' breasts", stating that their partner's breasts are "for my eyes only".
Similarly Harner et al. ${ }^{3}$ reported that teenage mothers expressed they were uncomfortable "to have my breast out like that [to breastfeed]". Furthermore, one teenage mother in their study explicitly expressed her partner's possessive attitude towards her breasts as well as fear of deprivation of his role in fatherhood: "he was afraid he wouldn't be able to feed her and he didn't want me to do it in public with everyone looking at me. ${ }^{3}$

Possessive attitudes toward breasts and fear of losing roles in the postnatal period contributed to the overarching theme of the lack of support from fathers to breastfeeding. Our study supports the findings of previous studies that in the setting of teenage pregnancy, the expectant fathers' support of breastfeeding (or lack of) will bea significant factor in the mother's decision-making process to breastfeed her infant. ${ }^{3,4,13}$

Breastfeeding as a natural process emerged as both negative and positive themes in our study. Some fathers looked favourably upon breastfeeding, regarding it as a natural and convenient process. This was in line with the general support for natural childbirth and analgesia free birth. It seems incongruous that, given the high regard for natural birthing processes, so many did not equally favour natural breastfeeding.

Lack of awareness of the benefits of breastfeeding in expectant fathers and mothers in the setting of teenage pregnancy has been previously reported to be another major contributor to the poor rate of breastfeeding. ${ }^{3,4}$ This lack of knowledge was displayed in themes emerging from expectant fathers with negative attitudes who stated the benefits of breastfeeding were a myth or expressed the attitude formula was better because milk volumes could be measured and the content of formula was somehow healthier for the baby.

Of note, few fathers in the setting of teenage pregnancy had undertaken more than 12years of education. This may simply reflect their younger age and resultant lack of opportunity to pursue further education. However, lower levels of education may be a contributing factor in the poor perceptions, attitudes and levels of knowledge.

Education to make expectant fathers in the setting of teenage pregnancy more aware of the science and benefits of breastfeeding may negate other fears and reservations. Sikorski et al. ${ }^{18}$ reported that health professional support for breastfeeding during the antenatal and postnatal period was a key component on the duration of breastfeeding. However, a previous randomised trial of a postnatal home visiting service by midwifes failed to achieve prolongation in breastfeeding outcomes, ${ }^{17}$ despite being a group we can predict may benefit from additional education. ${ }^{19}$

The consistency of negative and positive themes across previous studies and the present study highlight that the attitudes toward breastfeeding of fathers in the setting of teenage pregnancy have remained constant over the past decade despite the growth in education opportunities, and progressive perceptions on the role of fatherhood in modern society. One interesting factor is that no study, including our own, found fathers commenting on whether breastfeeding was "positive for the mother". The focus of comments across studies was on the father and baby to the exclusion of the mother. This may reflect the egocentric nature of adolescence or fathers deliberately ignoring comments about the mothers as the various studies were deemed to be "father's studies".

The advantages of this study are the comparison group of older 
fathers and mixed methodology. The limitations of the study are that results are confined to individuals recruited from a single metropolitan health service region and that attitudes were surveyed only on a single occasion in the third trimester. Attitudes may alter after delivery.

\section{Clinical implications}

The study indicates that many expectant fathers in the setting of teenage pregnancy may have a neutral or negative attitude towards breastfeeding. Strategies to promote breastfeeding that only target the expectant mother are likely to fail without engaging and educating the father. Therefore, support and education centred on breastfeeding should actively include fathers during the antenatal and postnatal periods.

\section{Conclusion}

The majority of teenage fathers had a neutral or negative attitude towards breastfeeding. The lack of endorsement from fathers is likely to influence persistence with breastfeeding in the setting of teenage pregnancy. The five negative themes emerged in this study may stem from lack of awareness of the short-term and long-term health benefits for infant and mother. Therefore breastfeeding education for fathers and enhanced support in the setting of a teenage pregnancy is desirable.

\section{Acknowledgements}

None.

\section{Competing interests}

The authors declare no competing interests in respect to this manuscript.

\section{Author's contributions}

JQ and RP undertook concept and design, ethics approvals and established the Australian Fathers Study. DL undertook data acquisition and undertook the first draft of the manuscript. JQ performed analysis. All authors interpreted the data and critically reviewed and subsequently approved the final manuscript for submission.

\section{References}

1. Lemay C, Cashman S, Elfenbein D, et al. A qualitative study of the meaning of fatherhood among young urban fathers. Public Health Nurs. 2010;27(3):221-31.

2. Hauck YL, Fenwick J, Dhaliwal SS, et al. A Western Australian survey of breastfeeding initiation, prevalence and early cessation patterns. $\mathrm{Ma}$ tern Child Health J. 2011;15(2):260-268.

3. Harner H, McCarter-Spaulding D. Teenage mothers and breastfeeding: Does paternal age make a difference? J Hum Lact. 2004;20(4):404-408.
4. Scott J, Binns C, Arnold R. Attitudes toward breastfeeding in Perth Australia: Qualitative analysis. Journal of Nutrition Education. 1997;29(5):244-249.

5. Fenwick J, Bayes S, Johansson M. A qualitative investigation into the pregnancy experiences and childbirth expectations of Australian fathers to be. Sex Reprod Health. 2012;3(1):3-9.

6. Lohan M, Cruise S, O'Halloran P, et al. Adolescent men's attitudes in relation to pregnancy and pregnancy outcomes: A systematic review of the literature from 1980-2009. J Adolesc Health. 2010;47(4):327-345.

7. Chin R, Hall P, Daiches A. Fathers' experiences of their transition to fatherhood: a metasynthesis. Journal of Reproductive and Infant Psychology. 2011;29(1):4-18.

8. Quinlivan J, Condon J. Anxiety and depression in fathers in teenage pregnancy. Aust N Z J Psychiatry. 2005;39(10):915-920.

9. Alio A, Mbah A, Grunsten R, et al. Teenage pregnancy and the influence of paternal involvement on fetal outcomes. Journal of Pediatric and Adolescent Gynaecology.2011;24(6):404-409.

10. Oddy W, Kendall G, Li K, et al. The long-term effects of breastfeeding on child and adolescent health: a pregnancy cohort study followed for 14years. J Pediatr. 2010;156(4):568-574.

11. Dyson L, McCormick F, Renfrew M. Interventions for promoting the initiation of breastfeeding. Cochrane Database of Systematic Reviews. 2005;8(2):CD001688

12. Hornell A, Lagstrom H, Lande B, et al. Breastfeeding, introduction of other foods and effects on health: a systematic literature review for 5th Nordic Nutrition recommendations. Food and Nutrition research. 2013;57:20823

13. Shah M, Gee R, Theall K. Partner support and impact of birth outcomes among teen pregnancy in the United States. J Pediatr Adolesc Gynecol. 2014;27(1):14-19.

14. Australian Bureau of Statistics Births 2012. Australia.

15. Elo S, Kyngäs $\mathrm{H}$. The qualitative content analysis process. $J$ Adv Nurs. 2008;62(1):107-115.

16. Yngve A, Sjostrom M. Breastfeeding determinants and a suggested framework for action in Europe. Public Health Nutr. 2001;4(28):729-739.

17. Quinlivan J, Box H, Evans S. Postnatal home visits in teenage mothers: a randomized controlled trial. Lancet. 2003;362(9361):893-900.

18. Sikorski J, Renfrew M, Pindoria S, et al. Support for breastfeeding mothers: a systemic review. Paediatric and Perinatal Epidemiology. $2003 ;(4): 407-417$.

19. Quinlivan J, Kua S, Gibson R, et al. Can we identify women who initiate and then prematurely cease breastfeeding? An Australian multicentre cohort study. International Breastfeeding Journal. 2015;10:16. 